\title{
Review
}

\section{Granzyme B cleavage of autoantigens in autoimmunity}

\author{
E Darrah ${ }^{1}$ and A Rosen ${ }^{*, 1}$
}

The systemic autoimmune diseases are a complex group of disorders characterized by elaboration of high titer autoantibodies and immune-mediated damage of tissues. Two striking features of autoimmune rheumatic diseases are their self-sustaining nature and capacity for autoamplification, exemplified by disease flares. These features suggest the presence of a feed-forward cycle in disease propagation, in which immune effector pathways drive the generation/release of autoantigens, which in turn fuel the immune response. There is a growing awareness that structural modification during cytotoxic granule-induced cell death is a frequent and striking feature of autoantigens, and may be an important principle driving disease. This review focuses on granzyme B (GrB)-mediated cleavage of autoantigens including (i) features of GrB cleavage sites within autoantigens, (ii) co-location of cleavage sites with autoimmune epitopes, and (iii) GrB sensitivity of autoantigens in disease-relevant target tissue. The mechanisms whereby GrB-induced changes in autoantigen structure may contribute to the initiation and propagation of autoimmunity are reviewed and reveal that GrB has the potential to create or destroy autoimmune epitopes. As there remains no direct evidence showing a causal function for GrB cleavage of antigens in the generation of autoimmunity, this review highlights important outstanding questions about the function of GrB in autoantigen selection.

Cell Death and Differentiation (2010) 17, 624-632; doi:10.1038/cdd.2009.197; published online 15 January 2010

\section{Features of Systemic Autoimmune Disease}

The systemic autoimmune diseases are a complex group of disorders, which include systemic lupus erythematosus (SLE), rheumatoid arthritis (RA), autoimmune myopathies, Sjögren's syndrome (SS), vasculitis, and scleroderma. The diseases manifest in a wide range of clinical phenotypes, often united by chronic inflammation and involvement of multiple organ systems. A dominant feature of these diseases is the elaboration of high titer, high affinity autoantibodies, which are strongly associated with phenotype. ${ }^{1-3}$ Although the clinical presentations of the individual systemic autoimmune diseases are often quite distinct, the presence of overlapping features in different diseases suggest the involvement of common effector pathways and shared disease mechanisms. ${ }^{4}$ This includes autoimmune myopathies occurring in myositis, SLE, and scleroderma, or the sicca complex (dry eyes/mouth) in many of the systemic autoimmune diseases. Autoantigens in systemic autoimmune diseases appear to be ubiquitously expressed molecules and may be central to the disease process, but the mechanisms underlying their selection remain unclear. Defining the features unifying these autoantigens, which may impact their selection by the immune response, is an important priority.

One striking feature of autoimmune rheumatic diseases is their self-sustaining nature, and their striking capacity for autoamplification (exemplified by disease flares). The autoantibody response in active disease is typically of high affinity and titer, and seems to be driven by ongoing antigen release. This amplification strongly suggests the presence of a feedforward cycle in disease propagation consisting of two key components: active immune effector pathways and immunogenic self-antigens. Immune effector pathways drive the generation/release of autoantigens from target tissue, which in turn provide fuel to propagate the immune response. Autoantigens are not passive players in this process, but rather have a direct function in shaping the autoimmune response. Identifying such amplifying pathways may have important therapeutic implications.

There is a growing awareness that structural changes of autoantigens during cytotoxic lymphocyte granule-mediated cell death are a frequent feature of systemic autoimmune diseases. Although this review focuses on structural changes of intracellular autoantigens induced by the granule component granzyme $B(\mathrm{GrB})$, it is important to note that there are several other mechanisms by which cytotoxic granules may contribute to the self-sustaining cycle of tissue damage in autoimmune disorders. First, structural modification of an overlapping yet distinct set of autoantigens has been shown for other members of the granzyme family including granzyme A $(\operatorname{GrA})^{5,6}$ and granzyme $\mathrm{H}(\mathrm{GrH}){ }^{7}$ Second, granzymes can

\footnotetext{
${ }^{1}$ Division of Rheumatology, Department of Medicine, Johns Hopkins University School of Medicine, Baltimore, MD, USA

*Corresponding author: A Rosen, Division of Rheumatology, Johns Hopkins University School of Medicine, Mason F. Lord Building, Center Tower, Room 412, Baltimore, MD 21224, USA. Tel: + 410550 1900; Fax: + 410550 2072; E-mail: arosen@jhmi.edu

Keywords: granzyme; autoimmunity; autoantibody; autoantigen; antigen processing; proteolysis

Abbreviations: SLE, systemic lupus erythematosus; RA, rheumatoid arthritis; SS, Sjögren's syndrome; GrB, granzyme B; GrA, granzyme A; GrH, granzyme H; APC, antigen-presenting cell; PM, polymyositis; lle, isoleucine; Val, valine; Leu, leucine; NLS, nuclear-localization signal; MG, myasthenia gravis; AchR, acetylcholine receptor; MCB, medullary carcinoma of the breast; MHC, major histocompatibility complex; AEP, asparaginyl endopeptidase; TTCF, tetanus toxoid C fragment; MBP, myelin basic protein; Dct, dopachrome tautomerase; HRS/Jo-1, histidyl-transfer RNA synthetase; HCC, hepatocellular carcinoma; GluR3, glutamate receptor subunit 3; NMR, nuclear magnetic resonance; DXMS, deuterium exchange mass spectrometry; PDC-E2, pyruvate dehydrogenase complex-E2; PBC, primary biliary cirrhosis Received 06.10.09; accepted 05.11.09; Edited by D Granville; published online 15.1.10
} 
mediate direct extracellular effects on target tissue. Neurotoxicity is induced after incubation of neurons with GrB through G-coupled protein receptor signaling and may have a function in the pathogenesis of neuroinflammatory diseases. ${ }^{8}$ In addition, degradation of extracellular matrix proteins by $\mathrm{GrB}$ is thought to have a function in cytotoxicity of smooth muscle cells in inflammatory vascular disease..$^{9,10}$ Finally, a function for granzymes as independent proinflammatory modulators of antigen-presenting cell (APC) function has been suggested by the finding that mouse macrophages treated with GrA secrete pro-inflammatory cytokines IL- $1 \beta$, TNF $\alpha$, and IL- 6 . $^{11}$ This review discusses the GrB-mediated changes in autoantigen structure and mechanisms whereby these changes may contribute to the initiation and propagation of autoimmunity.

\section{Granzymes Modify the Structure of Autoantigens during Cytolytic Granule-Mediated Cell Death}

During lymphocyte-induced cytotoxicity, granzymes and the pore-forming protein perforin act in concert to provide protease access to the target cell cytosol. Granzymes induce cell death by several processes, including activation of the caspase cascade, as well as direct cleavage of various intracellular substrates that participate in nuclear fragmentation and cell death. ${ }^{12}$ Significant data exists to suggest an important function for the cytotoxic lymphocyte granule pathway in the pathogenesis of systemic autoimmunity. Activated cytotoxic lymphocytes are enriched in the lung in scleroderma, ${ }^{13,14}$ the salivary gland in $S S,{ }^{15}$ and the synovium in RA. ${ }^{16}$ In the muscle of individuals with polymyositis (PM), T cells in direct contact with muscle fibers have been shown to orient components of the cytolytic granule pathway toward the site of cell-cell contact. ${ }^{17}$ Cytolytic CD8 + $T$ cells invading muscle fibers of PM patients exhibit a restricted $\mathrm{T}$-cell receptor repertoire suggesting clonal expansion of autoantigenic $T$ cells in the inflamed target tissue. ${ }^{18}$ Furthermore, increased circulating levels of cytolytic cells and components of the granule pathway correlate with disease activity. For example, the number of circulating activated cytolytic $\mathrm{CD} 8+\mathrm{T}$ cells correlates with disease activity in SLE, ${ }^{19}$ whereas the levels of GrB in the serum and synovial fluid of RA patients is strikingly associated with the severity of erosive joint disease. ${ }^{20,21}$

Multiple studies have shown that the majority of autoantigens targeted in systemic autoimmune diseases are substrates for granzymes, particularly $\mathrm{GrB}^{5,6,22-24}$ (Table 1). The association between $\mathrm{GrB}$ cleavability and autoantigen status is striking as, until recently, there have been few GrB substrates identified which are not autoantigens. A recent comprehensive proteomic analysis by Van Damme et al. ${ }^{25}$ revealed hundreds of novel GrB targets, and may suggest a pool of earlier undefined autoantigens.

The tetrapeptide substrate specificity of human GrB has been determined. In addition to the requirement for aspartic acid in the P1 position, P4 is specific for isoleucine (Ile), valine (Val), and leucine (Leu), whereas P3 and P2 are able to accommodate a wider range of amino acids ${ }^{26,27}$ (Figure 1a). Although there are many tetrapeptides satisfying the GrB consensus sequence within molecules, most of these sites are not cleaved by $\mathrm{GrB}^{28}$ Indeed, GrB typically cleaves autoantigen substrates at a limited number of sites. ${ }^{22}$ Interestingly, although $\mathrm{GrB}$ has similar tetrapeptide substrate specificity to the upstream group III caspases (which process effector caspases 3 and 7), ${ }^{27}$ distinct sites are used generating unique fragments of autoantigens not seen during caspase-mediated cell death. ${ }^{22}$

The GrB cleavage sites defined within autoantigens reveal several interesting features (Figure 1b). First, all have Ile, Leu, or $\mathrm{Val}$ in $\mathrm{P} 4$ and are cleaved after the $\mathrm{P} 1$ aspartic acid. ${ }^{22}$ Second, amino acids in the P2 and P3 positions in autoantigens are almost universally favored by human $\mathrm{GrB}$, but not tolerated by caspases (e.g. proline in P2). ${ }^{26}$ Finally, the native three-dimensional antigen structure may determine GrB cleavage site accessibility. For example, although $\mathrm{GrB}$ is able to cleave an in vitro generated autoantigen (SS transmembrane autoantigen, muscarinic receptor 3 ) at two distinct sites, only one site is used during granule-induced killing of intact human salivary gland target cells. ${ }^{23}$ These features reinforce the strong relationship between autoantigen status and this unique cleavage susceptibility.

Not unexpectedly, granzyme cleavage of antigens frequently has consequences in terms of function, ${ }^{5,24}$ including redistribution of nuclear antigens to the cytosol during cytotoxic granule-induced cytotoxicity. ${ }^{6,29}$ The SS nuclear autoantigen La/SSB is a substrate for both $\mathrm{GrB}$ and $\mathrm{GrH}$, and undergoes dramatic redistribution during granule-mediated cell death after uncoupling the C-terminal nuclear-localization signal (NLS). ${ }^{29}$ Similarly, cleavage of SLE and autoimmune hepatitis antigen lamin B by GrA and B causes disruption of the nuclear lamina, by uncoupling lamin B from its NLS. ${ }^{6}$ The granule pathway has also been shown to trigger the release of autoantigens from target cells. Blanco et al. ${ }^{19}$ showed that cytolytic CD8 + T cells from patients with active, but not quiescent, SLE were able to stimulate the release of nucleosomes from target cells, together with cleavage of nuclear autoantigens (e.g. U1-70 kDa). It is conceivable that cytoplasmically redistributed granzyme-cleaved antigens are released during granule-induced death, potentially influencing pathways of antigen presentation.

The ability of GrB to modify the structure of self-antigens is not limited to systemic autoimmunity. There are examples of tissue specific ${ }^{28,30}$ and cancer autoantigens being GrB substrates. $^{31}$ Myasthenia gravis (MG) is a tissue-specific autoimmune disease mediated by the antibody-dependent reduction of acetylcholine receptors (AchR) at the neuromuscular junction. ${ }^{32}$ Both $\mathrm{GrB}$ and its substrate (AchR $\varepsilon$ subunit) are aberrantly expressed in thymuses from patients with $M G$, suggesting that an immune response to AchR may propagate in the thymus. ${ }^{30}$ In medullary carcinoma of the breast (MCB), GrB-expressing cytotoxic T cells are found in large numbers, often nearby apoptotic tumor cells. Autoantibodies to $\beta$-actin have been identified in these patients and are able to recognize actin that has been redistributed to the surface of the apoptotic tumor cells. GrB cleavage fragments of $\beta$-actin have been detected in MCB tumor lysates, suggesting that CTL-mediated death of MCB tumor cells and actin redistribution may have a function in the generation of autoimmunity to $\beta$-actin. ${ }^{31}$ Interestingly, patients with MCB have a significantly higher survival rate than patients with non-MCB breast 
Table 1 Autoantigens cleaved by granzyme B have diverse functions and subcellular localizations

\begin{tabular}{|c|c|c|c|c|c|}
\hline Autoantigen & Accession No. & $\begin{array}{l}\text { Disease } \\
\text { association }\end{array}$ & Function & Localization & References \\
\hline Acetylcholine receptor $\varepsilon$ subunit $(A c h R \varepsilon)$ & NP_000071 & MG & Acetylcholine receptor & Plasma membrane & 30 \\
\hline$\beta$-actin & NP 001092 & MCB & Structural protein & Cytosol & 31 \\
\hline AHNAK & NP_-001611 & SLE & Calcium signaling & Cytosol/nucleus & 81 \\
\hline Alanyl tRNA synthetase (ARS) & NP_001596 & Myositis & Translation & Cytosol & 22 \\
\hline Centromere protein B (CENP-B) & NP_001801 & SSc & Mitosis & Nucleus & 22,73 \\
\hline Centromere protein C(CENP-C) & NP 001803 & SSc & Mitosis & Nucleus & 73 \\
\hline $\begin{array}{l}\text { Chromodomain helicase DNA binding } 4 \\
\text { (/CHD4/Mi-2) }\end{array}$ & NP_001264 & Myositis & Gene expression & Nucleus & 22 \\
\hline DNA-PK catalytic subunit (DNA-PKcs) & NP 008835 & Myositis & DNA repair & Nucleus & 22,24 \\
\hline Fibrillarin & NP_001427 & SSC & rRNA processing & Nucleolous & 22 \\
\hline$\alpha$-fodrin & NP_001123910 & SS & Cytoskeletal protein & Cytosol & 23 \\
\hline Glutamate receptor subunit 3 (GluR3) & NP_000819 & $\mathrm{RE}$ & Glutamate receptor & Plasma membrane & 28 \\
\hline $\begin{array}{l}\text { Human endogenous retrovirus } \mathrm{K}-10 \text { gag } \\
\text { (HERV-K10) }\end{array}$ & P87889 & SLE & Endogenous retrovirus & Nucleus & 82 \\
\hline Histidyl tRNA synthetase (HRS/Jo-1) & NP_002100 & Myositis & Translation & Cytosol & 22,46 \\
\hline Ki-67 & NP_002408 & Myositis & Proliferation & Nucleus & 22 \\
\hline $\mathrm{Ku}-70$ & NP_-001460 & Myositis, SLE & DNA repair & Nucleus & 22 \\
\hline $\mathrm{La} / \mathrm{SSB}$ & NP 003133 & SLE, SS & RNA binding & Nucleus & 22 \\
\hline Lamin B & NP_005564 & Hepatitis, SLE & Structural protein & Nuclear membrane & 6 \\
\hline Muscarinic acetylcholine receptor 3 (M3R) & NP 000731 & SS & Acetylcholine receptor & Plasma membrane & 23 \\
\hline Nuclear mitotic apparatus protein 1 (NuMA) & NP 006176 & SS & Mitosis & Nucleus & 22 \\
\hline Nucleolus organizing region $90 \mathrm{kDa}$ (NOR-90/UBF) & NP_055048 & SSc & Transcription factor & Nucleolous & 22 \\
\hline Nucleophosmin (B23) & NP_002511 & SSc & rRNA processing & Nucleolous & 47,48 \\
\hline Pyruvate dehydrogenase complex E2 (PDC-E2) & NP_001922 & PBC & Acetyl CoA synthesis & Mitochondria & 76 \\
\hline PMScl/EXOSC10 & NP_001001998 & $\begin{array}{l}\text { Myositis/SSc } \\
\text { overlap }\end{array}$ & mRNA degradation & Cytosol & 22 \\
\hline Poly(ADP)ribose polymerase 1 (PARP1) & NP_001609 & SLE & Ribosylation & Nucleus & 22 \\
\hline Postmeiotic segreqation 1 (PMS1) & NP 000525 & Myositis & DNA mismatch repair & Nucleus & 22 \\
\hline Postmeiotic segregation 2 (PMS2) & NP 000526 & Myositis & DNA mismatch repair & Nucleus & 22 \\
\hline RNA polymerase I (RNA Pol I) & NP_056240 & SSc & Transcription & Nucleus & 22 \\
\hline RNA polymerase II (RNA Pol II) & NP 000928 & SSc & Transcription & Nucleus & 22 \\
\hline Signal recognition particle $72 \mathrm{kDa}$ (SRP-72) & NP 008878 & Myositis, SLE & Translation & Cytosol & 22 \\
\hline Topoisomerase 1 (Topo-1) & NP_003277 & SSc & Transcription & Nucleus & 22 \\
\hline $\begin{array}{l}\text { U1 small nuclear ribonucleoprotein } 70 \mathrm{kDa} \\
\text { (U1-70 kDa) }\end{array}$ & NP_003080 & $\begin{array}{l}\text { Myositis, } \\
\text { SLE. SSc }\end{array}$ & RNA processing & Nucleus & 22 \\
\hline Úbiquitin fusion degradation 2 (UFD2) & NP_001099032 & SSc & Ubiquitination & Nucleus & 83 \\
\hline XRCC4 & NP_072044 & SLE & DNA repair & Nucleus & 84 \\
\hline
\end{tabular}

Abbreviations: MCB, medullary carcinoma of the breast; MG, myasthenia gravis; PBC primary biliary cirrhosis; RE, Rasmussen's encephalitis; SLE, systemic lupus erythematosus; SS, Sjögren's syndrome; SSc, scleroderma

cancer, suggesting that autoimmunity generated against tumor self-antigens may contribute to the favorable prognosis. $^{33,34}$

These data show that a group of molecules, which share little in common in terms of structure, function, or distribution, are unified by their susceptibility to cleavage by a highly specific and fastidious protease that has a major function in immune effector pathways. To place in context the possible relevance of GrB-mediated cleavage of autoantigens to initiation and propagation of autoimmunity, it is necessary to review the concepts of immunodominance and the ability of single, early proteolytic events to influence epitope selection.

\section{Protein Structure and Early Proteolytic Events Influence Epitope Selection during Antigen Presentation}

Structural modification of autoantigens including proteinprotein interactions, post-translational modifications, mutation of key residues, and differential sensitivity to proteases can lead to striking changes in the peptides that are ultimately presented to T cells. ${ }^{35}$ Molecules that are found in, or gain access to the extracellular space (including those derived from dying cells), are phagocytosed by professional APCs and gain access to the major histocompatibility complex (MHC) class II antigen processing pathway. On entry, antigens are exposed to a variety of proteases in the endosomal compartment, which process the native antigen into peptide fragments, available for loading onto class II molecules. Interestingly, although a large number of potential peptides can be generated, only a few peptides are successfully presented on the surface of the APC. These peptides are termed as 'immunodominant'. 36

There is accumulating evidence to suggest a function for protein conformation and $\mathrm{MHC}$-guided processing as one mechanism underlying immunodominance. ${ }^{37}$ During antigen processing, exposed structural determinants of native antigens can bind to MHC class II molecules and be protected from proteolysis by endosomal cathepsins, whereas the rest of the molecule is degraded. Alterations in antigen structure could, therefore, have profound effects on the regions of the protein available for binding to, and protection by, class II molecules. During development of immune tolerance, $T$ cells with high affinity for dominant peptides derived from selfproteins are often deleted or rendered non-responsive. ${ }^{38}$ 
a Group III Caspase
(Caspase-9)
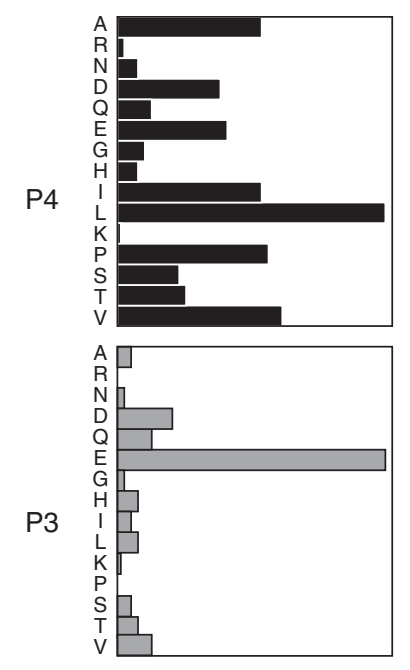

$A$
$R$
$N$
$N$
$D$
$Q$
E
G
G
$\quad H$

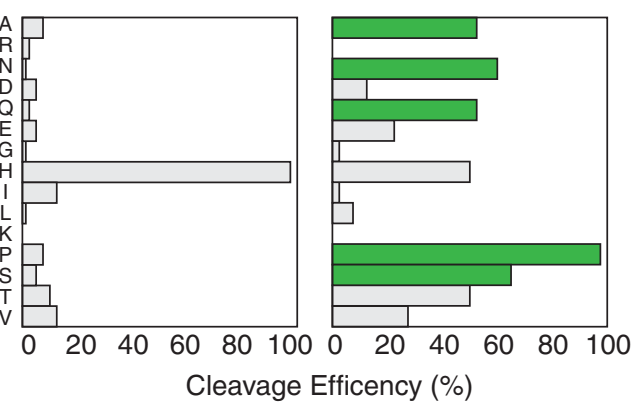

b

Autoantigen

Granzyme B

P4 P3 P2 P1

$\begin{array}{lllll}\text { AchRe } & \text { I } & D & \text { । } & D^{195}\end{array}$

ARS $\quad V \quad A \quad P D^{632}$

CENP-B $\quad \mathrm{V} \quad \mathrm{D} \quad \mathrm{S}^{457}$

CHD4/Mi-2 V $\quad D \quad P D^{1312}$

DNA-PKcs $V \quad G \quad P D^{2698}$

fibrillarin $\quad V \quad G \quad P \quad D^{184}$

GluR3 I S N D

HRS/Jo-1 L G $P D^{48}$

Ku-70 I $S \quad S \quad D^{79}$

La/SSB $\quad L \quad E \quad E D^{220}$

lamin $B \quad V \quad E \quad V \quad D^{231}$

M3R $M \quad D \quad Q D^{330}$

$\begin{array}{llll}P & S & S & D^{387}\end{array}$

NuMA $\quad \mathrm{A} \quad \mathrm{A} \quad \mathrm{T}^{1705}$

B23 $\quad V \quad E \quad E D^{122}$

L A $A D^{161}$

PMScl $\quad \mathrm{E} \quad \mathrm{Q}^{252}$

$\begin{array}{lllll}\text { PARP1 } & V & D & D^{536}\end{array}$

$\begin{array}{llll}\text { PMS1 I } & S & A^{496}\end{array}$

PMS2 $\quad V \quad E \quad K D^{493}$

RNA Pol II I T $P D^{370}$

$\begin{array}{lllll}\text { SRP-72 } & V & T & P & D^{573}\end{array}$

Topo-1 I E A D

U1-70kDa L G N D 409

Figure 1 Cleavage sites in autoantigens are enriched in amino acids specifically preferred by GrB over group III caspases. (a) Specificities of GrB and group III caspases are similar, but distinct. A comparison of the P2, P3, and P4 position requirements for a group III caspase (caspase-9) and GrB reveals differences in preferred amino acids (modified from Thornberry et $a^{26}$ ). (b) Cleavage sites of autoantigens known to be GrB substrates are enriched in amino acids that are preferred by GrB in the P2 and P3 positions over caspase-9. Amino acids preferred by GrB are shown in green for the P2 and in blue for the P3 positions. Amino acids are indicated as follows: A, alanine; R, arginine; N, asparagine; D, aspartic acid; Q, glutamine; E, glutamic acid; G, glycine; H, histidine; I, isoleucine; L, leucine; M, methionine; K, lysine; P, proline; S, serine; $T$, threonine; V, valine

Those peptides that are not presented on class II molecules at significant levels are termed as 'cryptic', and $\mathrm{T}$ cells recognizing such cryptic determinants are not tolerized and persist. Differences in antigen processing of self-proteins occurring as a result of structural modification could enhance cryptic epitope presentation, leading to activation of selfreactive $\mathrm{T}$ cells. ${ }^{36}$ Activation of $\mathrm{T}$ cells recognizing cryptic epitopes has been proposed to be an important mechanism underlying the development of autoimmunity. ${ }^{39}$

Recent studies showing the importance of early and highly specific proteolytic events in presentation of immunodominant and cryptic epitopes are highly relevant to the observation that autoantigens are proteolyzed in a novel way by granzymes. For example, asparaginyl endopeptidase (AEP) is a cysteine protease involved early in MHC class II antigen processing. Cleavage of intact endocytosed protein by AEP occurs at a limited number of asparagine residues, yet has profound effects on downstream processing by lysosomal proteases. ${ }^{40}$ Thus, cleavage of tetanus toxoid C fragment (TTCF) at a single asparagine residue by AEP is critical for presentation of multiple peptide epitopes derived from other regions of the protein. Mutation of the TTCF AEP cleavage site alters the pattern of fragments generated during subsequent processing and dramatically reduces activation of several antigenspecific T-cell hybridomas. ${ }^{41}$

Processing by AEP has been implicated in the generation of autoimmunity. For example, a major disease-associated epitope in the multiple sclerosis autoantigen myelin basic protein (MBP) contained within amino-acids 84-102 is normally cryptic, because of proteolysis at Asn94 by AEP. Inhibition of AEP activity in APCs enhances presentation of this epitope, which is a dominant epitope when autoimmunity to MBP is subsequently initiated. ${ }^{42}$ In addition, proteolytic activity of AEP is impaired when asparagine residues are $\mathrm{N}$-glycosylated, suggesting a way in which post-translational modifications can affect susceptibility to proteolysis and subsequent antigen presentation. ${ }^{40}$

In this regard, it is notable that antigen proteolysis and epitope selection is also influenced by subtle changes in antigen structure and conformation in several models of autoimmunity. For example, the post-translational conversion of aspartic acid to isoaspartyl residues is an isomerization that occurs naturally during cell stress, aging, and activation. The presence of isoaspartyl residues in full-length pigeon cyto- 
chrome $\mathrm{C}$ alters processing by cathepsin $\mathrm{D}$ and stimulates $\mathrm{T}$-cell proliferation. However, the aspartyl form is not stimulatory, suggesting that accumulation of a naturally occurring amino-acid modification can render self-antigens immunogenic. ${ }^{43}$ Immune responses to isoaspartyl, but not aspartyl, forms of the SLE autoantigen U1/Sm ribonucleoprotein have also been observed. Interestingly, activated B and T cells contain increased levels of isoaspartyl residues and may act as sources of autoantigen to drive the self-sustaining loop of autoimmunity. ${ }^{44}$ Furthermore, modification of single aminoacid residues within endogenous proteins can generate autoimmunity to cryptic epitopes. For example, dopachrome tautomerase (Dct) is an antigen normally expressed in melanocytes. When randomly mutated constructs of Dct are expressed in mice, autoimmunity to Dct is induced, resulting in skin hypopigmentation and activation of autoreactive $T$ cells recognizing cryptic Dct epitopes. Autoreactive $\mathrm{T}$ cells and hypopigmentation are also observed when the Dct constructs encoding truncated versions of the molecule, not generated during normal processing of the full-length protein, are expressed. ${ }^{45}$

Taken together, these studies show that relatively minor changes in autoantigen structure, including single proteolytic events, can have striking effects on epitope selection during antigen processing and presentation on MHC class II molecules. Autoimmunity may arise when normally dominant epitopes of self-antigens are destroyed or cryptic epitopes are revealed.

\section{Conformational State of Antigens Affects Susceptibility to GrB Cleavage}

As noted above, proteolysis and epitope selection can be affected by minor changes in antigen structure, including post-translational modifications, ${ }^{40}$ isomerizations, ${ }^{43}$ and early cleavage events. ${ }^{41}$ Several studies have shown that the tissue-specific expression ${ }^{46-48}$ or conformation ${ }^{49,50}$ of proteins can affect their susceptibility to cleavage by GrB. For example, GrB cleavage of extracellular matrix proteins (vitronectin and fibronectin) and the clotting mediator, von Willebrand factor, only occurs when the proteins are in distinct matrix-associated conformations. ${ }^{49,50}$ These studies suggest that autoantigens may be susceptible to GrB cleavage only under very specific circumstances associated with unique activation, differentiation, or mitotic states of the cell (Figure 2).

Granzyme-sensitive forms of autoantigens have been observed in the disease-relevant target tissue in myositis and scleroderma. For example, expression of a GrB-sensitive form of histidyl-transfer RNA synthetase (HRS/Jo-1), a target antigen in patients with interstitial lung disease associated with myositis, ${ }^{51}$ has also been noted exclusively in lung tissue as compared with other tissues including placenta, liver, muscle, ovary, and colon. The exclusive expression of granzyme-sensitive HRS/Jo-1 in the lung (which is the target of immune-mediated damage in this disease) is of interest. It is consistent with the proposal that GrB-mediated cleavage has a function in antigen selection, and that expression of cleavable autoantigens in specific target tissues may focus amplifying injury at that site. ${ }^{46} \mathrm{~A}$ similar tissue-specific

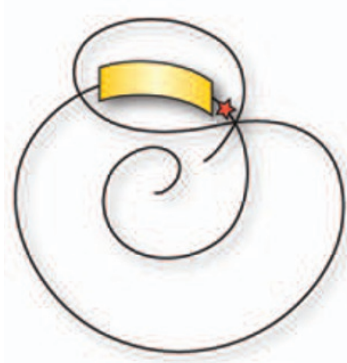

Ubiqutious conformation

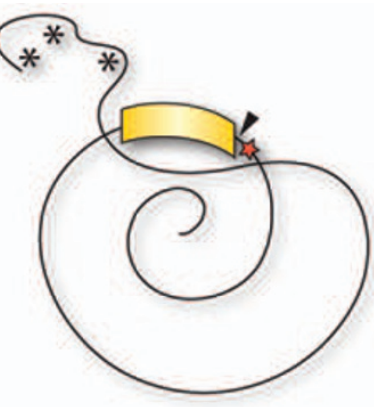

Tissue-specific conformation
Figure 2 Cleavage of GrB-sensitive forms of autoantigens may reveal cryptic epitopes in disease-relevant target tissue. GrB-sensitive forms of autoantigens may be present in the disease-relevant target tissue because of unique intermolecular interactions or post-translational modifications ( $\left(^{*}\right)$ exposing GrB cleavage sites (red star). Seemingly ubiquitously expressed autoantigens may be susceptible to $\operatorname{GrB}$ cleavage (black triangle) only when in tissue-specific immunogenic conformations and may drive the generation of autoimmunity to cryptic epitopes (yellow)

expression of a granzyme-sensitive form of the nucleolar autoantigen nucleophosmin (B23) has been observed in scleroderma. Autoantibodies to B23 are associated with the development of pulmonary hypertension in which vascular smooth muscle cell damage is believed to have a function in pathogenesis. ${ }^{52}$ Interestingly, B23 from differentiated, but not undifferentiated, vascular smooth muscle cells is efficiently cleaved by GrB. The restricted expression of cleavable autoantigens to particular cells in specific differentiation states within the target tissue suggests that either the property of cleavability or cleavage events themselves may be relevant to immune-mediated damage. ${ }^{47}$

B23 is also a target antigen in patients with hepatocellular carcinoma $(\mathrm{HCC}){ }^{53}$ Unlike normal regions of liver tissue, tumorous areas of liver also express a highly cleavable form of B23, which exists primarily as an SDS-stable oligomer. This cleavable form of B23 is preferentially recognized in HCC tumors by an antibody specifically raised to a peptide within the $\mathrm{GrB}$ cleavage site, suggesting that $\mathrm{HCC}$ liver expresses a form of B23 in which the GrB cleavage site is exposed, rendering it more sensitive to cleavage. ${ }^{48}$

Distinct or aberrant post-translational processing of autoantigens may also affect susceptibility to cleavage by GrB (Figure 2). The glutamate receptor subunit 3 (GluR3) is an autoantigen in the tissue-specific autoimmune disease Rasmussen's encephalitis. ${ }^{54}$ The glycosylation state of GluR3 seems to be critical in determining susceptibility to GrB cleavage. Although the $\mathrm{N}$-glycosylated granzyme-insensitive form predominates in neuronal tissue, a percentage is not glycosylated and is, therefore, susceptible to cleavage by GrB. It has been proposed that the balance of the glycosylated versus unglycosylated forms is modified during inflammation, potentially providing an opportunity for GrB-mediated cleavage only under very limited circumstances. ${ }^{28}$

Together, these data highlight that changes in protein conformation affect susceptibility to GrB cleavage in target tissues in autoimmunity as well as cancer. The association 
between status as an autoantigen and susceptibility to cleavage by $\mathrm{GrB}$ is striking, as are the clear examples in which the cleavable conformation of an autoantigen is enriched or expressed exclusively within the target tissue associated with an immune response to that antigen. Taken together, the data implicate novel cleavage events directed by immune effector pathways, occurring at specific tissue sites, as components of the pathways selecting self-antigens in autoimmune rheumatic diseases. It must be noted, however, that there is to date no direct evidence that GrB cleavage of autoantigens alters the immune response to that antigen, or participates in disease propagation. It is possible that cleavable forms of antigens exist in disease-relevant target tissues and represent an altered, immunogenic conformation that can drive the generation of autoimmunity even in the absence of GrB cleavage.

Unfortunately, both the tetrapeptide and the macromolecular specificities of human GrB differ significantly from mouse $\mathrm{GrB}$, and the unique unifying features around $\mathrm{GrB}$ cleavage sites in human autoantigens are largely unshared by mouse antigens. ${ }^{55-57}$ Defining the function of GrB in human systemic autoimmune diseases, therefore, depends on addressing the effects of $\mathrm{GrB}$ cleavage events on antigen immunogenicity in the human model - which constitutes a significant challenge. Possible ways around this barrier include: (i) examining effects of cleavage with human GrB (or fragments resulting from such cleavage) on immunogenicity of defined human autoantigens in mice; (ii) studying initiation and propagation of various forms of systemic autoimmunity in a GrB knock-out mouse or in a human GrB knock-in mouse; and (iii) directly testing effects of GrB on autoantigen immunogenicity and epitope selection in peripheral blood from human beings with autoimmune diseases in which $\mathrm{GrB}$ substrates are targeted.

Limited studies have been performed in this regard and suggest conflicting functions for GrB cleavage of antigens in the generation of autoimmunity. Mouse experiments have been performed, which suggest that fragments of antigens elicit more robust immune responses than intact protein. ${ }^{45,58}$ For example, $T$ cells from transgenic mice expressing human MHC class II molecules (DRB1*0301/DQB1*0201) associated with the development of immune responses to SLE autoantigen $\mathrm{La}$ exhibit enhanced T-cell proliferation on peptide re-challenge after priming with a truncated form of the protein. This naturally occurring frame-shift mutant (amino-acids 1-204) is very similar in length to the N-terminal fragment of La generated after GrB cleavage (amino-acids 1-220) suggesting that fragmentation of La may enhance antigen processing and release of cryptic epitopes. ${ }^{58}$ A study in GrB knock-out mice shows that GrB is not required for the development of pristane-induced SLE and may even have a protective effect. GrB knock-out mice injected with pristane exhibited features of SLE, including autoantibody production, but seemed to have increased mortality after pristane treatment, compared with wild-type mice. ${ }^{59}$ Experiments in human GrB knock-in mice may provide insights into the effect of autoantigen cleavage in a mouse model of autoimmunity, provided that the GrB cleavage site within the autoantigen is conserved between mice and human beings. It is clear that future studies are necessary to elucidate the potential function for GrB cleavage of autoantigens in the generation of autoimmunity and will require studies in the human model.

\section{GrB Cleavage Sites Reside in Unstructured Regions of Antigens and Co-locate with Autoimmune Epitopes}

The ability to obtain detailed information about the structure of autoantigens by crystallography as well as by dynamic methods including protein nuclear magnetic resonance (NMR) and deuterium exchange mass spectrometry may provide valuable information regarding the three-dimensional context of granzyme cleavage sites. Although this data is available for very few antigens at this time, some interesting themes are emerging. Analysis of the available crystal structures of autoantigens known to be cleaved by GrB reveals common features of granzyme cleavage sites. ${ }^{60,61}$ Partial structures of $B 23,{ }^{62}$ poly(ADP)ribose polymerase $1,{ }^{63}$ and HRS/Jo- $1^{64}$ show that GrB cleavage sites are located in unstructured regions or in areas adjacent to highly structured elements such as coiled coils. Similarly, the GrB site (IEAD $\left.{ }^{15}\right)$ in the scleroderma antigen and DNA replication protein topoisomerase $\mathrm{I}$ is located in the highly extended 174 amino-acid $\mathrm{N}$-terminal domain. This region was determined by circular dichroism to be virtually completely unfolded. ${ }^{65}$ In other cases, known granzyme cleavage sites are located on loosely ordered surface loops or linker regions between structural elements. The structure of scleroderma autoantigen fibrillarin reveals that the GrB cleavage site $\left(V_{G P D}{ }^{184}\right.$ ) is located on a surface loop between an $\alpha$-helix and $\beta$-strand. ${ }^{66}$

The effect of antigen structure on epitope selection is highlighted by work from Landry ${ }^{67}$, which showed that disordered regions of antigens lead to increased presentation of epitopes from adjacent, structurally organized sequences. As GrB cleavage sites tend to be located in disordered areas or linker regions connecting structural elements, it is possible that cleavage of autoantigens at the GrB site influences the presentation of downstream or hidden structured epitopes (Figure 3). Again, a complete dataset is not available, but review of cleavage sites as well as known B- and T-cell epitopes of autoantigens reveals that granzymes and the immune system target overlapping regions of these proteins (Figure 4). Epitope mapping of La has revealed overlapping $\mathrm{B}^{68}$ and $\mathrm{T}$-cell ${ }^{58,69}$ epitopes in a region spanning the GrB cleavage site. Similarly, overlapping $\mathrm{B}^{70}$ and $\mathrm{T}$-cell ${ }^{71}$ epitopes have been identified in the SLE autoantigen $\mathrm{U} 1-70 \mathrm{kDa}$ in a C-terminal region containing the $\mathrm{GrB}$ cleavage site.

Although the discussion of the effect of autoantigen cleavage by granzymes has focused on antigen processing and presentation of peptide epitopes to T cells, it is clear that autoantibody epitopes can also be affected. Granzyme cleavage of autoantigens can lead to the creation of immunogenic protein fragments, which are preferentially recognized by autoantibodies from patients with systemic autoimmune diseases. ${ }^{72,73}$ Autoantibodies from some patients with primary SS have been shown to preferentially recognize GrB cleavage fragments of La/SSB. A subset of these patients has autoantibodies that recognize cryptic 
a
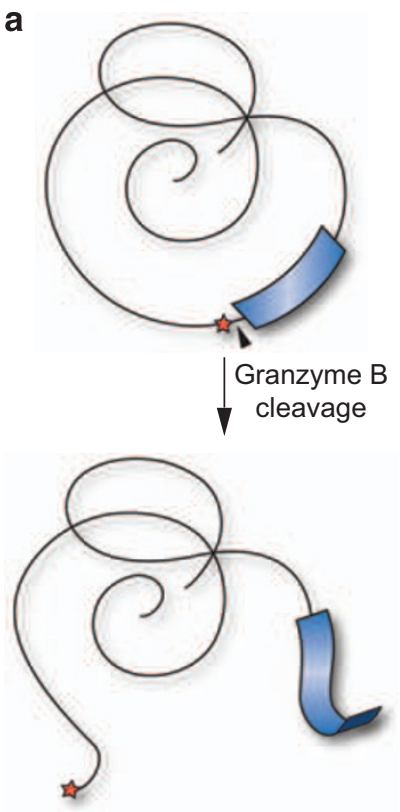

b

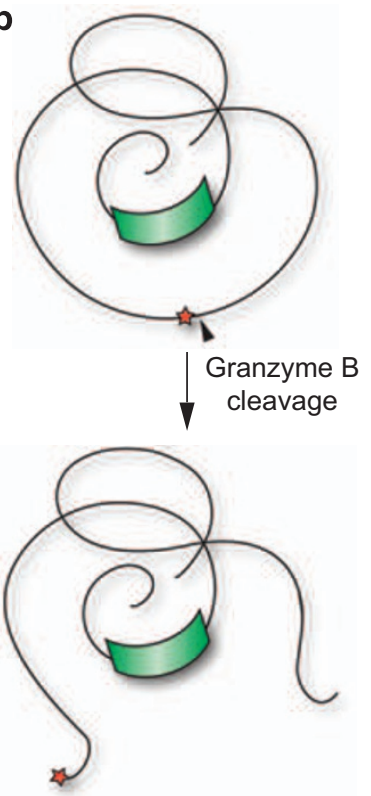

Figure 3 Cleavage of autoantigens at GrB cleavage sites located in unstructured loops may liberate cryptic epitopes derived from structural elements. (a) Proteolysis by GrB (black triangle) occurring in unstructured loops or linker regions of autoantigens (red star), may enhance presentation of cryptic epitopes derived from adjacent structural elements (blue). (b) GrB cleavage may also induce structural changes leading to increased presentation of cryptic epitopes derived from earlier hidden regions of autoantigens (green)

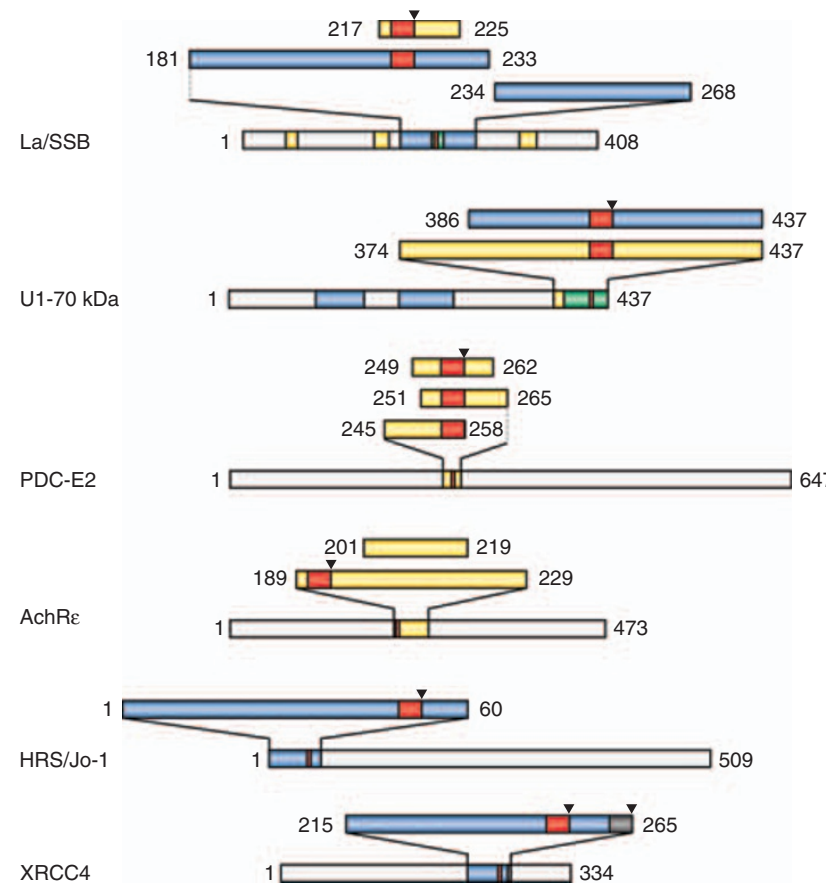

Figure 4 Autoimmune epitopes are co-located with GrB cleavage sites. B (blue) and $T$ (yellow) cell epitopes of several autoantigens are clustered in regions containing GrB cleavage sites (red). Overlapping B- and T-cell epitopes (green) in $\mathrm{La}$ and $\mathrm{U} 1-70 \mathrm{kDa}$ suggest autoimmune 'hot spots' in these regions of antigens containing GrB cleavage sites

epitopes generated after $\mathrm{GrB}$ cleavage, in that they cannot be blocked by intact La protein. ${ }^{72}$ Likewise, sera from patients with scleroderma and ischemic digital loss exhibit a striking preference for GrB-generated fragments of antigens, specifically those with autoantibodies to centromere protein CENP-C. ${ }^{73}$

Although cleavage of autoantigens by granzymes can aid in the generation of antigenic epitopes, granzyme cleavage also has the capacity to destroy immunoreactive epitopes. Circulating autoantibodies ${ }^{74}$ and $T$ cells $\mathrm{s}^{75}$ targeting pyruvate dehydrogenase complex-E2 (PDC-E2) are a frequent finding in patients with primary biliary cirrhosis (PBC). Although cleavage of PDC-E2 by caspases is capable of generating immunogenic fragments, GrB cleavage seems to destroy a dominant autoantibody epitope, abolishing reactivity by PBC patient sera. ${ }^{76}$ Interestingly, although two CD8 + T-cell epitopes may be destroyed by GrB cleavage ${ }^{77,78}$ another ${ }^{79}$ seems to be unaffected and perhaps even liberated by proteolysis. A similar pattern is observed for MG autoantigen AchRå, in which GrB cleavage has the potential to destroy a T-cell epitope while liberating another. ${ }^{80}$

\section{Conclusions}

There is strong evidence implicating the cytotoxic lymphocyte granule pathway in the pathogenesis of systemic autoimmune diseases. Activated cytotoxic lymphocytes are present in target tissues and effector function is positively correlated with disease activity in several diseases (e.g. myositis, SLE, RA). Autoantigens have diverse structures, functions, and subcellular localizations, yet are efficiently cleaved by GrB at a small number of unique sites, distinct from caspase cleavage sites. GrB-sensitive conformations of antigens have been detected in the disease-relevant target tissue in systemic autoimmune diseases. Analysis of available data shows that $\mathrm{GrB}$ cleavage sites are often situated in unstructured regions of antigens, adjacent to structured domains. Immune epitopes are also frequently located in close proximity to GrB cleavage sites, suggesting that cleavage by GrB may modulate presentation of epitopes from adjacent structured regions. Although there is clear data that single proteolytic events early during antigen processing can have striking effects on the epitopes presented, such data is not yet directly available for GrB. The observation that human and mouse GrB have distinct cleavage site specificities underscores the challenges to show a direct function for GrB-mediated cleavage of autoantigens in human autoimmunity. Studies in the human system will be needed, and will require an antigen, which is frequently targeted in a phenotypically distinct autoimmune disease, a substrate for human GrB, and associated with known MHC class II alleles. Establishing a mechanistic function for granzymes in the self-amplifying pathway of autoimmunity would have important therapeutic implications.

\section{Conflict of interest}

The authors declare no conflict of interest. 
1. Gabrielli A, Svegliati S, Moroncini G, Avvedimento EV. Pathogenic autoantibodies in systemic sclerosis. Curr Opin Immunol 2007; 19: 640-645.

2. Gunawardena H, Betteridge ZE, McHugh NJ. Myositis-specific autoantibodies: their clinical and pathogenic significance in disease expression. Rheumatology (Oxford) 2009; 48: 607-612.

3. Migliorini P, Baldini C, Rocchi V, Bombardieri S. Anti-Sm and anti-RNP antibodies. Autoimmunity 2005; 38: 47-54.

4. Rodriguez-Reyna TS, Alarcon-Segovia D. Overlap syndromes in the context of shared autoimmunity. Autoimmunity 2005; 38: 219-223.

5. Zhu P, Martinvalet D, Chowdhury D, Zhang D, Schlesinger A, Lieberman J. The cytotoxic T lymphocyte protease granzyme $A$ cleaves and inactivates poly(adenosine $5^{\prime}$-diphosphateribose) polymerase-1. Blood 2009; 114: 1205-1216.

6. Zhang D, Beresford PJ, Greenberg AH, Lieberman J. Granzymes A and B directly cleave lamins and disrupt the nuclear lamina during granule-mediated cytolysis. Proc Natl Acad Sci USA 2001; 98: 5746-5751.

7. Romero V, Fellows $E$, Jenne DE, Andrade F. Cleavage of La protein by granzyme $H$ induces cytoplasmic translocation and interferes with La-mediated HCV-IRES translational activity. Cell Death Differ 2009; 16: 340-348.

8. Wang T, Allie R, Conant K, Haughey N, Turchan-Chelowo J, Hahn K et al. Granzyme B mediates neurotoxicity through a G-protein-coupled receptor. FASEB J 2006; 20: 1209-1211.

9. Choy JC, Hung VH, Hunter AL, Cheung PK, Motyka B, Goping IS et al. Granzyme B induces smooth muscle cell apoptosis in the absence of perforin: involvement of extracellular matrix degradation. Arterioscler Thromb Vasc Biol 2004; 24: 2245-2250.

10. Boivin WA, Cooper DM, Hiebert PR, Granville DJ. Intracellular versus extracellular granzyme B in immunity and disease: challenging the dogma. Lab Invest 2009; 89: $1195-1220$

11. Metkar SS, Menaa C, Pardo J, Wang B, Wallich R, Freudenberg M et al. Human and mouse granzyme A induce a proinflammatory cytokine response. Immunity 2008; 29: 720-733.

12. Chowdhury D, Lieberman J. Death by a thousand cuts: granzyme pathways of programmed cell death. Annu Rev Immunol 2008; 26: 389-420.

13. Boin F, Wigley FM, Schneck JP, Oelke M, Rosen A. Evaluation of topoisomerase-1-specific CD8+ T-cell response in systemic sclerosis. Ann N Y Acad Sci 2005; 1062: 137-145.

14. Luzina IG, Atamas SP, Wise R, Wigley FM, Choi J, Xiao HQ et al. Occurrence of an activated, profibrotic pattern of gene expression in lung CD8+ T cells from scleroderma patients. Arthritis Rheum 2003; 48: 2262-2274.

15. Fujihara T, Fujita H, Tsubota K, Saito K, Tsuzaka K, Abe T et al. Preferential localization of CD8+ alpha $E$ beta $7+T$ cells around acinar epithelial cells with apoptosis in patients with Sjogren's syndrome. J Immunol 1999; 163: 2226-2235.

16. Namekawa T, Wagner UG, Goronzy JJ, Weyand CM. Functional subsets of CD4T cells in rheumatoid synovitis. Arthritis Rheum 1998; 41: 2108-2116.

17. Goebels N, Michaelis D, Engelhardt M, Huber S, Bender A, Pongratz D et al. Differential expression of perforin in muscle-infiltrating $\mathrm{T}$ cells in polymyositis and dermatomyositis. J Clin Invest 1996; 97: 2905-2910.

18. Bender A, Ernst N, Iglesias A, Dornmair K, Wekerle H, Hohlfeld R. T-cell receptor repertoire in polymyositis: clonal expansion of autoaggressive CD8+ T cells. J Exp Med 1995; 181 1863-1868

19. Blanco P, Pitard V, Viallard JF, Taupin JL, Pellegrin JL, Moreau JF. Increase in activated CD8+ T lymphocytes expressing perforin and granzyme $B$ correlates with disease activity in patients with systemic lupus erythematosus. Arthritis Rheum 2005; 52: 201-211.

20. Tak PP, Spaeny-Dekking L, Kraan MC, Breedveld FC, Froelich CJ, Hack CE. The levels of soluble granzyme $A$ and $B$ are elevated in plasma and synovial fluid of patients with rheumatoid arthritis (RA). Clin Exp Immunol 1999; 116: 366-370.

21. Goldbach-Mansky R, Suson S, Wesley R, Hack CE, El-Gabalawy HS, Tak PP. Raised granzyme $\mathrm{B}$ levels are associated with erosions in patients with early rheumatoid facto positive rheumatoid arthritis. Ann Rheum Dis 2005; 64: 715-721.

22. Casciola-Rosen L, Andrade F, Ulanet D, Wong WB, Rosen A. Cleavage by granzyme B is strongly predictive of autoantigen status: implications for initiation of autoimmunity. $J$ Exp Med 1999; 190: 815-826.

23. Nagaraju K, Cox A, Casciola-Rosen L, Rosen A. Novel fragments of the Sjogren's syndrome autoantigens alpha-fodrin and type 3 muscarinic acetylcholine receptor generated during cytotoxic lymphocyte granule-induced cell death. Arthritis Rheum 2001; 44: 2376-2386.

24. Andrade F, Roy S, Nicholson D, Thornberry N, Rosen A, Casciola-Rosen L. Granzyme B directly and efficiently cleaves several downstream caspase substrates: implications fo CTL-induced apoptosis. Immunity 1998; 8: 451-460.

25. Van Damme P, Maurer-Stroh S, Plasman K, Van Durme J, Colaert N, Timmerman E et al. Analysis of protein processing by $\mathrm{N}$-terminal proteomics reveals novel species-specific substrate determinants of granzyme B orthologs. Mol Cell Proteomics 2009; 8: 258-272.

26. Thornberry NA, Rano TA, Peterson EP, Rasper DM, Timkey T, Garcia-Calvo M et al. A combinatorial approach defines specificities of members of the caspase family and granzyme B. Functional relationships established for key mediators of apoptosis. J Biol Chem 1997; 272: 17907-17911.

27. Harris JL, Peterson EP, Hudig D, Thornberry NA, Craik CS. Definition and redesign of the extended substrate specificity of granzyme B. J Biol Chem 1998; 273: 27364-27373.
28. Gahring L, Carlson NG, Meyer EL, Rogers SW. Granzyme B proteolysis of a neuronal glutamate receptor generates an autoantigen and is modulated by glycosylation. $J$ Immunol 2001; 166: 1433-1438.

29. Huang M, Ida H, Arima K, Nakamura H, Aramaki T, Fujikawa $\mathrm{K}$ et al. La autoantigen translocates to cytoplasm after cleavage during granzyme B-mediated cytotoxicity. Life Sci 2007; 81: 1461-1466.

30. Casciola-Rosen L, Miagkov A, Nagaraju K, Askin F, Jacobson L, Rosen A et al. Granzyme B: evidence for a role in the origin of myasthenia gravis. J Neuroimmunol 2008; 201-202: 33-40.

31. Hansen MH, Nielsen $\mathrm{H}$, Ditzel HJ. The tumor-infiltrating B cell response in medullary breast cancer is oligoclonal and directed against the autoantigen actin exposed on the surface of apoptotic cancer cells. Proc Natl Acad Sci USA 2001; 98: 12659-12664.

32. Drachman DB. Myasthenia gravis. N Engl J Med 1994; 330: 1797-1810.

33. Ridolfi RL, Rosen PP, Port A, Kinne D, Mike V. Medullary carcinoma of the breast: a clinicopathologic study with 10 year follow-up. Cancer 1977; 40: 1365-1385.

34. Rapin V, Contesso G, Mouriesse H, Bertin F, Lacombe MJ, Piekarski JD et al. Medullary breast carcinoma. A reevaluation of 95 cases of breast cancer with inflammatory stroma. Cancer 1988; 61: 2503-2510.

35. Hall JC, Casciola-Rosen L, Rosen A. Altered structure of autoantigens during apoptosis. Rheum Dis Clin North Am 2004; 30: 455-471, vii.

36. Sercarz EE, Lehmann PV, Ametani A, Benichou G, Miller A, Moudgil K. Dominance and crypticity of T cell antigenic determinants. Annu Rev Immunol 1993; 11: 729-766.

37. Sercarz EE, Maverakis E. Mhc-guided processing: binding of large antigen fragments. Nat Rev Immunol 2003; 3: 621-629.

38. Singh NJ, Schwartz RH. Primer: mechanisms of immunologic tolerance. Nat Clin Pract Rheumatol 2006; 2: 44-52.

39. Lanzavecchia A. How can cryptic epitopes trigger autoimmunity? J Exp Med 1995; 181: 1945-1948.

40. Manoury B, Hewitt EW, Morrice N, Dando PM, Barrett AJ, Watts C. An asparaginyl endopeptidase processes a microbial antigen for class II MHC presentation. Nature 1998; 396: 695-699.

41. Antoniou AN, Blackwood SL, Mazzeo D, Watts C. Control of antigen presentation by a single protease cleavage site. Immunity 2000; 12: 391-398.

42. Manoury B, Mazzeo D, Fugger L, Viner N, Ponsford M, Streeter H et al. Destructive processing by asparagine endopeptidase limits presentation of a dominant T-cell epitope in MBP. Nat Immunol 2002; 3: 169-174.

43. Doyle HA, Gee RJ, Mamula MJ. Altered immunogenicity of isoaspartate containing proteins. Autoimmunity 2007; 40: 131-137.

44. Mamula MJ, Gee RJ, Elliott JI, Sette A, Southwood S, Jones PJ et al. Isoaspartyl posttranslational modification triggers autoimmune responses to self-proteins. J Biol Chem 1999; 274: 22321-22327.

45. Engelhorn ME, Guevara-Patino JA, Noffz G, Hooper AT, Lou O, Gold JS et al. Autoimmunity and tumor immunity induced by immune responses to mutations in self. Nat Med 2006; 12: 198-206

46. Levine SM, Raben N, Xie D, Askin FB, Tuder R, Mullins M et al. Novel conformation of histidyl-transfer RNA synthetase in the lung: the target tissue in Jo-1 autoantibodyassociated myositis. Arthritis Rheum 2007; 56: 2729-2739.

47. Ulanet DB, Flavahan NA, Casciola-Rosen L, Rosen A. Selective cleavage of nucleolar autoantigen B23 by granzyme B in differentiated vascular smooth muscle cells: insights into the association of specific autoantibodies with distinct disease phenotypes. Arthritis Rheum 2004; 50: 233-241.

48. Ulanet DB, Torbenson M, Dang CV, Casciola-Rosen L, Rosen A. Unique conformation of cancer autoantigen B23 in hepatoma: a mechanism for specificity in the autoimmune response. Proc Natl Acad Sci USA 2003; 100: 12361-12366.

49. Buzza MS, Zamurs L, Sun J, Bird CH, Smith Al, Trapani JA et al. Extracellular matrix remodeling by human granzyme $\mathrm{B}$ via cleavage of vitronectin, fibronectin, and laminin. $J$ Biol Chem 2005; 280: 23549-23558.

50. Buzza MS, Dyson JM, Choi H, Gardiner EE, Andrews RK, Kaiserman D et al. Antihemostatic activity of human granzyme B mediated by cleavage of von Willebrand factor. J Biol Chem 2008; 283: 22498-22504

51. Bernstein RM, Morgan SH, Chapman J, Bunn CC, Mathews MB, Turner-Warwick M et al. Anti-Jo-1 antibody: a marker for myositis with interstitial lung disease. Br Med J (Clin Res Ed) 1984; 289: 151-152.

52. Ulanet DB, Wigley FM, Gelber AC, Rosen A. Autoantibodies against B23, a nucleolar phosphoprotein, occur in scleroderma and are associated with pulmonary hypertension. Arthritis Rheum 2003; 49: 85-92.

53. Imai H, Ochs RL, Kiyosawa K, Furuta S, Nakamura RM, Tan EM. Nucleolar antigens and autoantibodies in hepatocellular carcinoma and other malignancies. Am J Pathol 1992; 140: 859-870.

54. Rogers SW, Andrews PI, Gahring LC, Whisenand T, Cauley K, Crain B et al. Autoantibodies to glutamate receptor GluR3 in Rasmussen's encephalitis. Science 1994; 265: 648-651.

55. Adrain C, Murphy BM, Martin SJ. Molecular ordering of the caspase activation cascade initiated by the cytotoxic T lymphocyte/natural killer (CTL/NK) protease granzyme B. J Biol Chem 2005; 280: 4663-4673.

56. Kaiserman D, Bird CH, Sun J, Matthews A, Ung K, Whisstock JC et al. The major human and mouse granzymes are structurally and functionally divergent. J Cell Biol 2006; 175 : $619-630$ 
57. Casciola-Rosen L, Garcia-Calvo M, Bull HG, Becker JW, Hines T, Thornberry NA et al. Mouse and human granzyme $B$ have distinct tetrapeptide specificities and abilities to recruit the bid pathway. J Biol Chem 2007; 282: 4545-4552.

58. Dudek NL, Maier S, Chen ZJ, Mudd PA, Mannering SI, Jackson DC et al. T cell epitopes of the La/SSB autoantigen in humanized transgenic mice expressing the HLA class II haplotype DRB1*0301/DQB1 ${ }^{*} 0201$. Arthritis Rheum 2007; 56: 3387-3398.

59. Graham KL, Thibault DL, Steinman JB, Okeke L, Kao PN, Utz PJ. Granzyme B is dispensable for immunologic tolerance to self in a murine model of systemic lupus erythematosus. Arthritis Rheum 2005; 52: 1684-1693.

60. Chen J, Anderson JB, DeWeese-Scott C, Fedorova ND, Geer LY, He S et al. MMDB: Entrez's 3D-structure database. Nucleic Acids Res 2003; 31: 474-477.

61. Wang Y, Addess KJ, Chen J, Geer LY, He J, He S et al. MMDB: annotating protein sequences with Entrez's 3D-structure database. Nucleic Acids Res 2007; 35 (Database issue): D298-D300.

62. Lee HH, Kim HS, Kang JY, Lee BI, Ha JY, Yoon HJ et al. Crystal structure of human nucleophosmin-core reveals plasticity of the pentamer-pentamer interface. Proteins 2007; 69: $672-678$.

63. Nagashima T, Hayashi F, Yokoyama S. Solution structure of Wgr domain of poly(AdpRibose) polymerase-1. Riken Structural Genomics Proteomics Initiative (RSGI). Deposition date: 2005. PDB ID: 2CR9.

64. Nameki N, Sasagawa A, Tomizawa T, Koshiba S, Inoue M, Kigawa T et al. Solution structures of the Whep-Trs domain of human histidyl-trna synthetase. Riken Structura Genomics Proteomics Initiative (RSGI). Deposition date: 2005. PDB ID: 1X59.

65. Stewart L, Ireton GC, Parker LH, Madden KR, Champoux JJ. Biochemical and biophysical analyses of recombinant forms of human topoisomerase I. J Biol Chem 1996; 271 : 7593-7601.

66. Min J, Wu H, Zeng $\mathrm{H}$, Loppnau $\mathrm{P}$, Weigelt J, Sundstrom $\mathrm{M}$ et al. Human Fibrillarin. Structural Genomics Consortium (SGC). Deposition date: 2006. PDB ID: 2IPX

67. Landry SJ. Local protein instability predictive of helper T-cell epitopes. Immunol Today 1997; 18: 527-532.

68. Rauh AJ, Hornig $\mathrm{H}$, Luhrmann R. At least three distinct $\mathrm{B}$ cell epitopes reside in the C-terminal half of La protein, as determined by a recombinant DNA approach. Eur $J$ Immunol 1988; 18: 2049-2057.

69. Davies ML, Taylor EJ, Gordon C, Young SP, Welsh K, Bunce M et al. Candidate T-cell epitopes of the human La/SSB autoantigen. Arthritis Rheum 2002; 46: 209-214.

70. Cram DS, Fisicaro N, Coppel RL, Whittingham S, Harrison LC. Mapping of multiple B-cell epitopes on the 70-kilodalton autoantigen of the U1 ribonucleoprotein complex. $\mathrm{J}$ Immunol 1990; 145: 630-635.

71. O'Brien RM, Cram DS, Coppel RL, Harrison LC. T-cell epitopes on the 70-kDa protein of the (U1)RNP complex in autoimmune rheumatologic disorders. J Autoimmun 1990; 3: 747-757.

72. Huang M, Ida H, Kamachi M, Iwanaga N, Izumi Y, Tanaka F et al. Detection of apoptosisspecific autoantibodies directed against granzyme B-induced cleavage fragments of the
SS-B (La) autoantigen in sera from patients with primary Sjogren's syndrome. Clin Exp Immunol 2005; 142: 148-154

73. Schachna L, Wigley FM, Morris S, Gelber AC, Rosen A, Casciola-Rosen L. Recognition of Granzyme B-generated autoantigen fragments in scleroderma patients with ischemic digital loss. Arthritis Rheum 2002; 46: 1873-1884

74. Van de Water J, Fregeau D, Davis P, Ansari A, Danner D, Leung P et al. Autoantibodies of primary biliary cirrhosis recognize dihydrolipoamide acetyltransferase and inhibit enzyme function. J Immunol 1988; 141: 2321-2324.

75. Shimoda S, Miyakawa $\mathrm{H}$, Nakamura M, Ishibashi $\mathrm{H}$, Kikuchi $\mathrm{K}$, Kita $\mathrm{H}$ et al. CD4 T-cell autoreactivity to the mitochondrial autoantigen PDC-E2 in AMA-negative primary biliary cirrhosis. J Autoimmun 2008; 31: 110-115.

76. Matsumura S, Van De Water J, Kita H, Coppel RL, Tsuji T, Yamamoto K et al. Contribution to antimitochondrial antibody production: cleavage of pyruvate dehydrogenase complex-E2 by apoptosis-related proteases. Hepatology 2002; 35: $14-22$.

77. Shimoda S, Nakamura M, Ishibashi H, Hayashida K, Niho Y. HLA DRB4 0101-restricted immunodominant $T$ cell autoepitope of pyruvate dehydrogenase complex in primary biliary cirrhosis: evidence of molecular mimicry in human autoimmune diseases. J Exp Med 1995; 181: $1835-1845$.

78. Matsumura S, Kita H, He XS, Ansari AA, Lian ZX, Van De Water J et al. Comprehensive mapping of HLA-A0201-restricted CD8 T-cell epitopes on PDC-E2 in primary biliary cirrhosis. Hepatology 2002; 36: 1125-1134.

79. Kita H, Lian ZX, Van de Water J, He XS, Matsumura S, Kaplan M et al. Identification of HLA-A2-restricted CD8(+) cytotoxic $T$ cell responses in primary biliary cirrhosis: $T$ cell activation is augmented by immune complexes cross-presented by dendritic cells. $J$ Exp Med 2002; 195: 113-123.

80. Hill M, Beeson D, Moss $\mathrm{P}$, Jacobson L, Bond A, Corlett L et al. Early-onset myasthenia gravis: a recurring T-cell epitope in the adult-specific acetylcholine receptor epsilon subunit presented by the susceptibility allele HLA-DR52a. Ann Neurol 1999; 45 224-231.

81. Skoldberg F, Ronnblom L, Thornemo M, Lindahl A, Bird PI, Rorsman F et al. Identification of AHNAK as a novel autoantigen in systemic lupus erythematosus. Biochem Biophys Res Commun 2002; 291: 951-958

82. Beyer TD, Kolowos W, Dumitriu IE, Voll RE, Heyder P, Gaipl US et al. Apoptosis of the teratocarcinoma cell line Tera-1 leads to the cleavage of HERV-K10gag proteins by caspases and/or granzyme B. Scand J Immunol 2002; 56: 303-309.

83. Mahoney JA, Odin JA, White SM, Shaffer D, Koff A, Casciola-Rosen L et al. The human homologue of the yeast polyubiquitination factor Ufd2p is cleaved by caspase 6 and granzyme B during apoptosis. Biochem J 2002; 361 (Pt 3): 587-595.

84. Lee KJ, Dong X, Wang J, Takeda Y, Dynan WS. Identification of human autoantibodies to the DNA ligase IV/XRCC4 complex and mapping of an autoimmune epitope to a potential regulatory region. J Immunol 2002; 169: 3413-3421. 